\title{
Review: "Reproducing GW150914: the first observation of gravitational waves from a binary black hole merger"
}

\author{
Ian Hawke ${ }^{1}$ \\ ${ }^{1}$ Affiliation not available
}

February 1, 2021

This is a review of manuscript CiSESI-2020-10-0108 submitted to the CiSE Reproducible Research Track: Reproducing GW150914: the first observation of gravitational waves from a binary black hole merger (Duncan Brown, Karan Vahi, Michela Taufer, Von Welch, Ewa Deelman).

\section{Content Summary}

A detailed reproduction of the LIGO collaboration's first gravitational wave detection.

\section{Recommendation}

The article should be accepted, although I recommend three substantive and a few minor modifications.

\section{Detailed review}

This article and the linked code give a detailed reproduction of the first claimed detection of gravitational waves through a binary black hole merger. The challenges are outlined in detail. All code and data is available through stable links (the importance of which is clearly demonstrated in the paper, as certain discrepancies are linked to slightly different data usage, and other steps are no longer available through the LIGO repositories).

This article was posted on the arxiv at https://arxiv.org/abs/2010.07244. The arxiv link did not use the arxiv Labs "Links to Code" feature, but all code and data file links are given in the paper.

As part of this review I ran the scripts on local resources and checked which steps worked. The amount of computing time needed to run the scripts on local resources is greater than the time available for the review, but I am confident that, with time, the full figure could be reproduced from the material provided here.

\section{Suggested modifications}

1. The paper references successful reproductions of the LIGO results, including this specific detection. However, no reference is made to, or discussion given of, the papers claiming null results based on the LIGO data (which, in this context, could be seen as unsuccessful reproductions), such as the papers 
of Jackson and collaborators (e.g., https://doi.org/10.1088/1475-7516/2016/08/029). I believe that in the context of reproduction and replication these papers should be referenced and briefly discussed.

2. The generate_workflow.sh script fails if run on a system without Python version 2, which is now the default on some systems (one of my checks was on a clean install of Ubuntu 20.04). This should be made clear in the appropriate README at least, and preferably in the Software Versions section of the paper. I would also recommend that the virtualenv calls in generate_workflow.sh and make_pycbc_hist.sh explicitly point to Python 2 (using -p python2), and that explicit Python calls in the latter script also explicitly point to Python 2.

3. The arXiV version contains links to all the appropriate repositories in footnotes. The version available through the ScholarOne system has removed these footnotes, and the links don't appear to have been replaced in the bibliography. I would suggest that the URLs/DOIs are put in the reference list and all footnotes (in the arXiV version) are replaced with cites as appropriate.

4. Some of the astrophysics jargon could use glossing, particularly around page 3. For example, to the non-specialist, what are (a) multi-messenger counterparts, (b) matched filtering, (c) down-weighting the SNR of non-Gaussian events, (d) time and mass parameters?

5. Explain why the focus is on the reproduction of the PyCBC search.

6. Figure 3 is too busy (particularly the $\mathrm{x}$ axis labels) to be clear.

7. p9, left column, line 23: the word "easily" is doing a lot of work here, and I would recommend removing it.

\section{Typos and trivialities}

1. p4, left column, line 38: "and the the codes"

2. p6, left column, line 33: check space around "generate_workflow.sh"

3. p6, right column, line 37: check formatting of reference ("abbott et al/")

4. p8, right column, line 44: grammar of "a schedulers" 